\title{
Decoherence Dynamics in Low-Dimensional Cold Atom Interferometers
}

\section{Citation}

Burkov, A. A., M. D. Lukin, and Eugene Demler. 2007. “Decoherence Dynamics in LowDimensional Cold Atom Interferometers." Physical Review Letters 98 (20) (May 17). doi:10.1103/ physrevlett.98.200404.

\section{Published Version}

doi:10.1103/PhysRevLett.98.200404

\section{Permanent link}

http://nrs.harvard.edu/urn-3:HUL.InstRepos:27891678

\section{Terms of Use}

This article was downloaded from Harvard University's DASH repository, and is made available under the terms and conditions applicable to Other Posted Material, as set forth at http:// nrs.harvard.edu/urn-3:HUL.InstRepos:dash.current.terms-of-use\#LAA

\section{Share Your Story}

The Harvard community has made this article openly available.

Please share how this access benefits you. Submit a story.

\section{Accessibility}




\title{
Decoherence Dynamics in Low-Dimensional Cold Atom Interferometers
}

\author{
A. A. Burkov, M. D. Lukin, and Eugene Demler \\ Department of Physics, Harvard University, Cambridge, Massachusetts 02138, USA
}

(Received 5 January 2007; published 17 May 2007)

\begin{abstract}
We report on a study of the dynamics of decoherence of a matter-wave interferometer, consisting of a pair of low-dimensional cold atom condensates at finite temperature. We identify two distinct regimes in the time dependence of the coherence factor of the interferometer: quantum and classical. Explicit analytical results are obtained in both regimes. In particular, in the two-dimensional case in the classical (long time) regime, we find that the dynamics of decoherence is universal, exhibiting a power-law decay with an exponent, proportional to the ratio of the temperature to the Kosterlitz-Thouless temperature of a single 2D condensate. In the one-dimensional case in the classical regime we find a universal nonanalytic time dependence of decoherence, which is a consequence of the nonhydrodynamic nature of damping in 1D liquids.
\end{abstract}

PACS numbers: 03.75.Gg, 03.75.Lm, 05.30.Jp, 74.40.+k

Understanding quantum phases and phase transitions is a substantially more complex task than understanding their classical counterparts. In particular, in the quantum case equilibrium and nonequilibrium properties are inseparable and thus dynamical relaxation processes and dissipation may have a profound effect on the nature of equilibrium phases and phase transitions. Superfluid to insulator transition in disordered 2D superconducting films or Josephson junction arrays (JJA) [1] is probably the most prominent example. While it is often thought of as one of the most basic and fundamental quantum phase transitions, many aspects of it are still very poorly understood. In this context it appears interesting to investigate nonequilibrium phenomena in low-dimensional superfluid systems in a simpler setting than is offered by such highly complex systems as amorphous superconducting films or JJA. Cold atom systems seem to be especially well suited for such studies since they allow for an unprecedented control of the relevant experimental parameters and for direct, real-time measurements of phase coherence [2-4].

In this Letter we present a study of the dynamics of decoherence in a system of two low-dimensional (1D or 2D) cold atom condensates that are prepared from a single phase coherent condensate by splitting it, using an optical lattice or a radio-frequency-field-induced potential on an atom chip. It has been recently demonstrated by a number of groups [3] that it is possible to split a single condensate in such a way that the phase coherence between the two halves is initially well preserved. This phase coherent state is, however, a highly nonequilibrium one for the splitcondensate system. The system will then relax to thermal equilibrium over time, in which the condensates are completely incoherent and it is the dynamics of this decoherence process that we are interested in.

The problem of decoherence in cold atom condensates has been studied theoretically before by several authors [5]. Previous studies mostly focused on the single-mode approximation, which assumes that only the lowest-energy phase mode needs to be taken into account. This approximation is reasonable in three-dimensional condensates, but breaks down in low-dimensional systems, where one needs to take into account the whole continuum of hydrodynamic (low-energy) modes, responsible for the enhanced fluctuations [6] and the absence of conventional order. Quantum decoherence due to such continuum of modes has been explored in a recent work by Bistritzer and Altman [7]. In this Letter we extend their analysis to investigate both quantum and thermal decoherence. We also discuss the role of the "squeezing factor," determined by the finite splitting time.

At a given temperature $T$ we can divide the hydrodynamic modes into two groups: classical, with energies $\epsilon<$ $T$, and quantum, with $\epsilon>T$. The dynamics of each group of modes is governed by classical and quantum fluctuations correspondingly. As a consequence, we can also identify two distinct regimes in the time dependence of the decoherence process: quantum and classical. At short times, i.e., times, shorter than the inverse temperature $t<1 / T$, decoherence dynamics is dominated by the quantum modes and thermal fluctuations may be neglected. Since at short times the memory of the initial state of the split system is still preserved, the quantum decoherence process is strongly influenced by the nature of this initial state, which in turn is determined by the process, by which a single condensate is split into two. We adopt a simple model, in which during the splitting the system is assumed to be described by a Josephson Hamiltonian with a timedependent tunneling term $[8,9]$ :

$$
H=\frac{E_{c}}{2} N_{r}^{2}-E_{J} \cos (\theta) \approx \frac{E_{c}}{2} N_{r}^{2}+\frac{E_{J}}{2} \theta^{2},
$$

where $N_{r}=N_{1}-N_{2}$ is the relative number of atoms in the two condensates, which is conjugate to the relative phase $\left[\theta, N_{r}\right]=i$. The charging energy $E_{c}=d \mu / d N=\mu / N$, where $\mu$ is the chemical potential, taken to be the same in both condensates, and $E_{J}$ is the (time-dependent) tunneling 
energy. Equation (1) is the Hamiltonian of a harmonic oscillator with a characteristic frequency $\omega=\sqrt{E_{c} E_{J}}$ (we will be using $\hbar=k_{B}=1$ units henceforth). One can distinguish two stages of the splitting process [10]. While $\omega(t)>1 / \tau_{s}$, where $\tau_{s}$ is the characteristic time of the splitting, the splitting is approximately adiabatic and the ground state of Eq. (1) should be a good approximation for the actual state of the double-well system. Once $\omega(t)<$ $1 / \tau_{s}$, the splitting process is no longer adiabatic. We can then approximate this second stage of the splitting process as instantaneous. This is equivalent to saying that we approximate the state of the fully split system, when $E_{J}=$ 0 , by the ground state of Eq. (1) at the moment $t^{*}$ when the adiabaticity condition fails, i.e., when $\omega\left(t^{*}\right)=1 / \tau_{s}$. Then we obtain the following result for the relative number fluctuations of the initial state of the fully split system: $\Delta N_{r} \sim \sqrt{N / \mu \tau_{s}}$, where $N=\varrho V=\left(N_{1}+N_{2}\right) / 2$. This formula agrees well with the apparent value of $\Delta N_{r}$, which can be inferred from the measurements of Ref. [11]. The agreement becomes even better if we take into account the parabolic trap potential, which gives $\mu \sim N^{2 / 5}$ instead of $\mu \sim N$ for the box geometry. It is useful to represent the above result in terms of a squeezing factor $\xi$, defined as $1 / \xi=\Delta N_{r} / \sqrt{N}=1 / \sqrt{\mu \tau_{s}}$. Our analysis is valid for $\xi \gg$ 1 . When $\mu \tau_{s} \lesssim 1$ one should take $\xi \approx 1$. Our formula for $\Delta N_{r}$ thus interpolates between the limits of fast splitting $\left(\tau_{s} \lesssim 1 / \mu\right)$, when $\Delta N_{r} \sim \sqrt{N}$, and slow (adiabatic) splitting, in which case $\Delta N_{r} \sim 0$.

Choosing the initial state of the system to be a minimum uncertainty wave packet of width $1 / \Delta N_{r}$, we find that the coherence factor, defined as $\Psi_{d}(t)=$ $(1 / V) \operatorname{Re} \int d \mathbf{x}\left\langle e^{i \theta(\mathbf{x}, t)}\right\rangle$, where $\theta(\mathbf{x}, t)$ is the relative phase between the two condensates, $V=L^{d}$ is the $d$-dimensional volume of each condensate $(d=1,2)$, and the angular brackets denote both quantum and thermal averages, is given by

$\Psi_{d}(t) \propto \exp \left[-\mu^{2} t^{2} / 2 N \xi^{2}\right] \begin{cases}\exp \left[-\mu t / 2 \pi \mathcal{K} \xi^{2}\right], & d=1, \\ \left(t / t_{0}\right)^{-\mu / 16 T_{\mathrm{KT}} \xi^{2}}, & d=2,\end{cases}$

where $t_{0} \sim 1 / \mu$ is a short-time cutoff. The KosterlitzThouless temperature $T_{\mathrm{KT}}$ of a single $2 \mathrm{D}$ condensate and the Luttinger parameter $\mathcal{K}$ in the $1 \mathrm{D}$ case are defined explicitly below. In the fast-splitting regime $\left(\tau_{s} \lesssim 1 / \mu\right)$ in 1D our result agrees with the result of Bistritzer and Altman [7].

At long times $t \gg 1 / T$ classical modes, with energies $\epsilon<T$, dominate the dynamics. In this case we find

$$
\Psi_{d}(t) \propto \begin{cases}\exp \left[-\left(t / t_{0}\right)^{2 / 3}\right], & d=1, \\ \left(t / t_{0}\right)^{-T / 8 T_{\mathrm{KT}},} & d=2,\end{cases}
$$

where $t_{0}$ are (dimension-dependent) cutoff times [time dependences in Eq. (3) are valid when $t>t_{0}$ ], given explicitly below. Two features are noteworthy here. In the 1D case classical decoherence dynamics has a nonanalytic time dependence. As shown below, this is a consequence of the fact that in 1D liquids damping at finite temperatures is always nonhydrodynamic; i.e., the damping rate is a nonanalytic function of momentum [12]. In both 1D and 2D cases the time dependence of decoherence in the classical regime turns out to be universal, independent of the microscopic nature of damping and interactions. This can make our result especially useful for thermometry in 2D condensates.

We now provide the most important details of the derivation of the above results. We consider a system of two 1D or $2 \mathrm{D}$ superfluids, which are prepared at time $t=0$ in a phase coherent state by, for example, splitting a single superfluid in a double-well optical potential. This highly nonequilibrium state will then relax to thermal equilibrium. We will assume that this relaxation is entirely due to intrinsic processes in each of the two superfluids. We start from the following imaginary time action for the double-well system:

$$
\begin{aligned}
S= & \int_{0}^{1 / T} d \tau \int d \mathbf{x}\left[\Phi_{\sigma}^{*}\left(\partial_{\tau}-\mu-\frac{\nabla^{2}}{2 m}\right) \Phi_{\sigma}\right. \\
& \left.+\frac{g}{2}\left|\Phi_{\sigma}\right|^{4}-J \Phi_{\sigma}^{*} \tau_{\sigma \sigma^{\prime}}^{x} \Phi_{\sigma^{\prime}}\right] .
\end{aligned}
$$

Here $\sigma=1,2$ labels the two condensates (summation over repeated indices is implicit) and $J$ is the residual tunneling matrix element between the condensates. We will assume henceforth that $J$ is negligible and set it to zero. Following Popov [13], we rewrite the action using density-phase variables, which we define as $\Phi_{\sigma}=\sqrt{\varrho}_{\sigma} e^{i \theta_{\sigma}}$, and expand about the uniform equal density saddle point $\varrho_{1}=\varrho_{2}=$ $\varrho=\mu / g$ to obtain the following hydrodynamic imaginary time action:

$$
\begin{aligned}
S= & S_{0}+S_{1}, \\
S_{0}= & \int_{0}^{1 / T} d \tau \int d \mathbf{x}\left[i h_{\sigma} \partial_{\tau} \theta_{\sigma}+\frac{g}{2} h_{\sigma}^{2}+\frac{\left(\boldsymbol{\nabla} h_{\sigma}\right)^{2}}{8 m \varrho}\right. \\
& \left.+\frac{\varrho\left(\nabla \theta_{\sigma}\right)^{2}}{2 m}\right], \\
S_{1}= & \int_{0}^{1 / T} d \tau \int d \mathbf{x} \frac{h_{\sigma}\left(\boldsymbol{\nabla} \theta_{\sigma}\right)^{2}}{2 m},
\end{aligned}
$$

where $h_{\sigma}=\varrho_{\sigma}-\varrho$. The saddle point expansion is legitimate at sufficiently low temperatures, such that the (exponentially small) contribution of phase slips (1D) or vortices (2D) to correlations can be neglected. The harmonic part of the action $S_{0}$ describes undamped collective density-phase modes of the double-well superfluid. The anharmonic part of the action $S_{1}$ is responsible for the dissipation and relaxation to equilibrium at finite temperatures, as will be shown below [13,14]. As discussed above, at short times $t<1 / T$ after the decoupling of the two condensates, the contribution of thermal fluctuations of the relative phase to decoherence dynamics is negligible and temperature can simply be set to zero: the dynamics of 
decoherence in this case is purely quantum. Furthermore, we may neglect the anharmonic terms in Eq. (5), which in the quantum regime are unimportant. Introducing the relative and center-of-mass phase variables $\theta=\theta_{1}-\theta_{2}, \chi=$ $\left(\theta_{1}+\theta_{2}\right) / 2$ and integrating over density fluctuations, we obtain

$$
\begin{aligned}
S & =S_{\chi}+S_{\theta}, \\
S_{\chi} & =\int_{0}^{1 / T} d \tau \int d \mathbf{x}\left[\frac{1}{g}\left(\partial_{\tau} \chi\right)^{2}+\frac{\varrho}{m}(\nabla \chi)^{2}\right], \\
S_{\theta} & =\int_{0}^{1 / T} d \tau \int d \mathbf{x}\left[\frac{1}{4 g}\left(\partial_{\tau} \theta\right)^{2}+\frac{\varrho}{4 m}(\nabla \theta)^{2}\right] .
\end{aligned}
$$

The relative and center-of-mass phase dynamics are thus completely decoupled at short times. As the action for the relative phase is purely harmonic, the problem of the relative phase dynamics at short times may be solved exactly [7]. Passing from the imaginary time action for the long-wavelength relative phase modes Eq. (6) back to the Hamiltonian, and rewriting it in Fourier space, we obtain

$$
H=\sum_{\mathbf{k}}\left[g\left|\Pi_{\mathbf{k}}\right|^{2}+\frac{\varrho \mathbf{k}^{2}}{4 m}\left|\theta_{\mathbf{k}}\right|^{2}\right]
$$

where $\left[\theta_{\mathbf{k}}, \Pi_{\mathbf{k}^{\prime}}\right]=i \delta_{\mathbf{k k}^{\prime}}$.

Taking the initial state of the split-condensate system $\left|\Phi_{0}\right\rangle$ to be a minimum uncertainty wave packet, as discussed above, it is straightforward to evaluate the time evolution of the coherence factor [7]. One obtains

$$
\begin{aligned}
\Psi(t) & =\frac{1}{V} \operatorname{Re} \int d \mathbf{x}\left\langle\Phi_{0}\left|e^{i H t} e^{i \theta(\mathbf{x})} e^{-i H t}\right| \Phi_{0}\right\rangle \\
& =e^{-(1 / 2 V) \sum_{\mathbf{k}}\left\langle\left|\theta_{\mathbf{k}}\right|^{2}\right\rangle_{t}},
\end{aligned}
$$

where

$$
\begin{aligned}
\left\langle\left|\theta_{\mathbf{k}}\right|^{2}\right\rangle_{t} & \equiv\left\langle\left.\Phi_{0}\left|e^{i H t}\right| \theta_{\mathbf{k}}\right|^{2} e^{-i H t} \mid \Phi_{0}\right\rangle \\
& =\frac{\mu \tau_{s}}{\varrho}\left[\cos ^{2}\left(\epsilon_{\mathbf{k}} t\right)+\frac{1}{\epsilon_{\mathbf{k}}^{2} \tau_{s}^{2}} \sin ^{2}\left(\epsilon_{\mathbf{k}} t\right)\right],
\end{aligned}
$$

and $\epsilon_{\mathbf{k}}=\sqrt{g \varrho / m} k \equiv c_{s} k$. Evaluating Eq. (8) at times $t>$ $1 / \mu$, we obtain Eq. (2), with $T_{\mathrm{KT}}=\pi \varrho / 2 m$ and $\mathcal{K}=$ $(\pi / 2) \sqrt{\varrho / g m}$ (these are weak-coupling expressions for $T_{\mathrm{KT}}$ and $\mathcal{K}$, which are expected to be accurate at low temperatures). Note that in Eq. (2) we have explicitly separated the contribution of the $\mathbf{k}=0$ mode (the common factor), which will contribute separately from the $k>0$ continuum in a finite-size system. This is nothing but the "phase diffusion" [11], which is the only contribution that exists at long times in bulk systems.

We now extend the above theory to the classical, i.e., long time $t>1 / T$ limit. The character of the long time dynamics is determined by the low-energy, classical modes, with energies $\epsilon<T$. We start from the hydrodynamic action for density and phase fluctuations Eq. (5). Unlike in the quantum case, the anharmonic terms in the action turn out to be crucial, as will become clear below. Despite the fact that the source of relaxation is purely intrinsic in our case, it turns out to be possible, and very useful, to cast the problem into the form [15] of an "observable macroscopic variable" interacting with a "thermal bath" of microscopic degrees of freedom. This is possible thanks to the following observation. While in the imaginary time action Eq. (5) all degrees of freedom enter on a completely equal footing, the initial conditions for the relative and center-of-mass variables, introduced above, are drastically different. Indeed, we can formally model the process of splitting a single condensate into two by suddenly changing the value of the tunneling amplitude in Eq. (2) from a large value at times $t<0$ to zero at $t=0$. In hydrodynamic description this is equivalent to suddenly driving a large gap for the relative phase-density collective modes to zero. Center-of-mass modes, however, feel this change only weakly, through anharmonic terms in the hydrodynamic action. It thus seems reasonable to assume that the center-of-mass modes approximately remain in thermal equilibrium throughout the splitting and subsequent relaxation process [16]. We can then think of these degrees of freedom as forming a thermal bath. The relative density and phase modes, in contrast, are strongly affected by the separation process and are far out of equilibrium at $t=0$. We thus arrive at the picture of out-of-equilibrium relative phase collective modes, interacting with a thermal bath of the center-of-mass modes, the interaction being described by the anharmonic terms in Eq. (5). To make this separation of the degrees of freedom explicit we can perturbatively integrate out the center-of-mass degrees of freedom (we expect this perturbation theory to work well at long times) in Eq. (5), and obtain the following effective harmonic action for the relative phase variables only:

$$
\begin{aligned}
S= & \int_{0}^{1 / T} d \tau \sum_{\mathbf{k}}\left[\frac{1}{4 g}\left|\partial_{\tau} \theta(\mathbf{k}, \tau)\right|^{2}+\frac{\varrho \mathbf{k}^{2}}{4 m}|\theta(\mathbf{k}, \tau)|^{2}\right] \\
& +\int_{0}^{1 / T} d \tau_{1} d \tau_{2} \sum_{\mathbf{k}} \Pi\left(\mathbf{k}, \tau_{1}-\tau_{2}\right) \theta\left(\mathbf{k}, \tau_{1}\right) \theta\left(-\mathbf{k}, \tau_{2}\right) .
\end{aligned}
$$

In $2 \mathrm{D}$ the lowest order (single phonon bubble) approximation for the dissipative kernel $\Pi(\mathbf{k}, i \omega)$ is sufficient $[13,17]$. In 1D the contribution of a single bubble diagram diverges "on shell," i.e., at $i \omega=\epsilon_{\mathbf{k}}$, which is a consequence of kinematics in 1D, namely, the fact that the laws of energy and momentum conservation are satisfied simultaneously [12]. It is then necessary to resum the most divergent (maximal number of bubbles) diagrams at each order [18]. We obtain

$$
\Pi(\mathbf{k}, i \omega)=\frac{|\omega|}{8 g} \begin{cases}\gamma_{1} \epsilon_{\mathbf{k}}^{3 / 2}, & d=1, \\ \gamma_{2} \epsilon_{\mathbf{k}}, & d=2,\end{cases}
$$

where $\gamma_{1}=\sqrt{2 \alpha} \overline{\mathcal{K}} / \pi \mu(\alpha \approx 1.954$ is a numerical constant) and $\gamma_{2}=4 \pi T^{2} / 3 \sqrt{3} \mu T_{\mathrm{KT}}$ are damping coefficients, characterizing the strength of dissipation. Note 
that while $\gamma_{2}$ is dimensionless, $\gamma_{1}$ has dimensions of $1 / \sqrt{\epsilon}$. The most interesting feature of Eq. (11) is the nonanalytic dependence of $\Pi(\mathbf{k}, i \omega)$ on $\mathbf{k}$ in the 1D case [12], which was first noted in a different context by Andreev. This means that damping in a 1D liquid at finite temperatures is nonhydrodynamic, which is a consequence of the breakdown of superfluid order in 1D on length scales longer than the temperature-dependent correlation length [12]. It is this nonanalytic dependence of the damping kernel on momentum that leads to the nonanalytic time dependence of decoherence in Eq. (3).

Using Keldysh formalism, it is possible to show that the imaginary time action Eq. (10) is exactly equivalent to the following real-time quantum Langevin equation $[19,20]$ :

$$
\begin{aligned}
& \frac{d^{2} \theta(\mathbf{k}, t)}{d t^{2}}+\epsilon_{\mathbf{k}}^{2} \theta(\mathbf{k}, t)+8 g \int_{-\infty}^{t} d t^{\prime} \Pi_{I}\left(\mathbf{k}, t-t^{\prime}\right) \theta\left(\mathbf{k}, t^{\prime}\right) \\
& \quad=2 g \zeta(\mathbf{k}, t)
\end{aligned}
$$

where the quantum noise variable $\zeta(\mathbf{k}, t)$ is defined by its autocorrelation function $\left\langle\zeta\left(\mathbf{k}, t_{1}\right) \zeta\left(-\mathbf{k}, t_{2}\right)\right\rangle=$ $2 \Pi_{R}\left(\mathbf{k}, t_{1}-t_{2}\right)$ and the functions $\Pi_{R, I}$ are given by

$$
\begin{aligned}
\Pi_{I}(\mathbf{k}, \omega) & =i \operatorname{Im} \Pi(\mathbf{k}, i \omega \rightarrow \omega+i 0+), \\
\Pi_{R}(\mathbf{k}, \omega) & =i \Pi_{I}(\mathbf{k}, \omega) \operatorname{coth}(\omega / 2 T) .
\end{aligned}
$$

Solving Eq. (12) with the initial conditions $\theta(\mathbf{k}, 0)=0$, $\dot{\theta}(\mathbf{k}, 0)=0$ [21], by the Laplace transform, we obtain

$$
\left\langle\theta^{2}(\epsilon, t)\right\rangle_{d}=\frac{T \mu}{\varrho \epsilon^{2}}\left[1-f_{d}(\epsilon, t)\right]
$$

where

$$
\begin{aligned}
& f_{1}(\epsilon, t)=e^{-\gamma_{1} \epsilon^{3 / 2} t}\left[1-\frac{\gamma_{1}^{2} \epsilon}{2} \sin ^{2}(\epsilon t)+\frac{\gamma_{1} \sqrt{\epsilon}}{2} \sin (2 \epsilon t)\right], \\
& f_{2}(\epsilon, t)=e^{-\gamma_{2} \epsilon t}\left[1-\frac{\gamma_{2}^{2}}{2} \sin ^{2}(\epsilon t)+\frac{\gamma_{2}}{2} \sin (2 \epsilon t)\right] .
\end{aligned}
$$

Note that in the limit $t \rightarrow \infty$ Eq. (14) correctly reproduces the equilibrium magnitude of thermodynamic fluctuations of $\theta(\epsilon)$. Now we can easily evaluate the time dependence of the coherence factor. At long times we obtain

$$
\Psi_{d}(t)=\exp \left[-\frac{1}{2} \int_{0}^{T} d \epsilon \nu_{d}(\epsilon)\left\langle\theta^{2}(\epsilon, t)\right\rangle_{d}\right],
$$

where $\nu_{d}(\epsilon)$ is the phonon density of states in a $d$-dimensional superfluid. Evaluating the integral over $\epsilon$, we arrive at Eq. (3), with the cutoff times given by

$$
t_{0}= \begin{cases}\beta \pi \mu \mathcal{K} / T^{2}, & d=1, \\ 1 / \gamma_{2} T, & d=2,\end{cases}
$$

where $\beta=\left[8 /(2 \alpha)^{1 / 3} \Gamma(1 / 3)\right]^{3 / 2} \approx 2.61$.

We acknowledge useful discussions with I. Affleck, E. Altman, I. Bloch, V. Gritsev, B. I. Halperin, W. Ketterle, M. Oberthaler, A. Polkovnikov, S. Sachdev, and J. Schmiedmayer. Financial support was provided by the National Science Foundation under Grants
No. DMR05-41988 and No. DMR01-32874, the HarvardMIT CUA, and AFOSR.

[1] M. P. A. Fisher, Phys. Rev. Lett. 65, 923 (1990); A. F. Hebard and M. A. Paalanen, ibid. 65, 927 (1990); H. S. J. van der Zant et al., ibid. 69, 2971 (1992).

[2] M. R. Andrews et al., Science 275, 637 (1997).

[3] Y. Shin et al., Phys. Rev. Lett. 92, 050405 (2004); T. Schumm et al., Nature Phys. 1, 57 (2005); R. Gati et al., Appl. Phys. B 82, 207 (2006).

[4] A. Polkovnikov et al., Proc. Natl. Acad. Sci. U.S.A. 103, 6125 (2006); Z. Hadzibabic et al., Nature (London) 441, 1118 (2006).

[5] M. Lewenstein and L. You, Phys. Rev. Lett. 77, 3489 (1996); J. Javanainen and S. M. Yoo, ibid. 76, 161 (1996); E. M. Wright et al., ibid. 77, 2158 (1996); J. Javanainen and M. Wilkens, ibid. 78, 4675 (1997); Y. Castin and J. Dalibard, Phys. Rev. A 55, 4330 (1997); G. J. Milburn et al., ibid. 55, 4318 (1997); C. Menotti et al., ibid. 63, 023601 (2001); I. Zapata et al., ibid. 67, 021603 (2003); A. Mebrahtu et al., ibid. 73, 033601 (2006).

[6] D. Hellweg et al., Appl. Phys. B 73, 781 (2001); S. Dettmer et al., Phys. Rev. Lett. 87, 160406 (2001); D. L. Luxat and A. Griffin, Phys. Rev. A 67, 043603 (2003).

[7] R. Bistritzer and E. Altman, arXiv:cond-mat/0609047.

[8] I. Zapata et al., Phys. Rev. A 57, R28 (1998).

[9] This analysis is done for the $\mathbf{k}=0$ mode. One can apply the same arguments for all modes with no change in the final result.

[10] A. J. Leggett and F. Sols, Phys. Rev. Lett. 81, 1344 (1998).

[11] G.-B. Jo et al., Phys. Rev. Lett. 98, 030407 (2007).

[12] A. F. Andreev, Sov. Phys. JETP 51, 1038 (1980); K. V. Samokhin, J. Phys. Condens. Matter 10, L533 (1998); D. S. Petrov et al., Phys. Rev. Lett. 84, 2551 (2000); D. S. Petrov et al., ibid. 85, 3745 (2000).

[13] V. N. Popov, Theor. Math. Phys. (Engl. Transl.) 11, 478 (1972).

[14] P. C. Hohenberg and P. C. Martin, Ann. Phys. (N.Y.) 34, 291 (1965).

[15] A. O. Caldeira and A. J. Leggett, Phys. Rev. Lett. 46, 211 (1981).

[16] In reality some degree of heating of these modes will occur. One should then think of the temperature $T$ in our equations as the temperature of the final equilibrium state of the system.

[17] S. T. Belyaev, Sov. Phys. JETP 7, 289 (1958).

[18] We expect our result for 1D to apply in the large $\mathcal{K}$ limit. In the Tonks-Girardeau (small $\mathcal{K}$ ) limit the bubble resummation leads to incorrect results. See M. Pustilnik et al., Phys. Rev. Lett. 96, 196405 (2006) and R. G. Pereira et al., ibid. 96, 257202 (2006).

[19] A. Schmid, J. Low Temp. Phys. 49, 609 (1982); A. O. Caldeira and A. J. Leggett, Physica (Amsterdam) 121A, 587 (1983); U. Eckern et al., Phys. Rev. B 30, 6419 (1984).

[20] K. Damle et al., Phys. Rev. A 54, 5037 (1996).

[21] We expect them to be a good approximation to the exact initial conditions in the long time limit. 